602.

\title{
ON THE POTENTIALS OF POLYGONS AND POLYHEDRA.
}

[From the Proceedings of the London Mathematical Society, vol. vi. (1874-1875), pp. 20-34. Read December 10, 1874.]

THE problem of the attraction of polyhedra is treated of by Mehler, Crelle, t. Lxvi. pp. 375-381 (1866); but the results here obtained are exhibited under forms, which are very different from his and which give rise to further developments of the theory.

\section{General Formula for the Potentials of a Cone and a Shell.}

1. The law of attraction is taken to be according to the inverse square of the distance; and I commence with the general case of a cone standing upon any portion of a surface $\Sigma$ as its base, and attracting a point at its vertex, the cone being considered as a mass of density unity.

2. Considering, in the first instance, an element of mass, the position of which is determined by its distance $r$ from the vertex (or origin) and by two angular coordinates defining the position of the radius vector $r$, then the element is $=r^{2} d r d \omega$ (where $d \omega$ is the element of solid angle, or surface of the unit-sphere), and the corresponding element of potential is $\frac{1}{r} r^{2} d r d \omega,=r d r d \omega$; whence

$$
V=\int r d r d \omega
$$

which, integrating from $r=0$ to $r=$ its value at the surface, is

$$
=\frac{1}{2} \int r^{2} d \omega
$$

where $r$ now denotes the radius vector at a point of the surface, being, therefore, a given function of the two angular coordinates: and the remaining (double) integration 
is to be extended to all values of the angular coordinates belonging to a position of $r$ within the conical surface which is the other boundary of the attracting mass, or say over the spherical aperture of the cone.

3. If the value of the radius vector at the surface is taken to be $m r(m$ a constant), then we have obviously

$$
V=\frac{1}{2} m^{2} \int r^{2} d \omega
$$

and hence also, writing $m+d m$ instead of $m$, we obtain, for the potential of the portion of the shell lying between the similar and similarly situated surfaces $\Sigma$ and $\Sigma^{\prime}$, belonging to the parameters $m$ and $m+d m$ respectively, the value

$$
V=m d m \int r^{2} d \omega
$$

this is $=2 \frac{d m}{m}$ into the potential of the cone; and we thus see that it is the same problem to determine the potential of the cone, and that of the subtended portion of the indefinitely thin shell included between the two surfaces.

4. The same result may be arrived at as follows: the element of solid angle $d \omega$ determines on the surface an element of surface $d \mathbf{\Sigma}$, and if $d \nu$ be the corresponding normal thickness of the shell, then the element of mass is $=d \nu d \Sigma$, and the element of potential is $=\frac{1}{m r} d \nu d \Sigma$ ( $m r$ being, as before, the radius vector at the surface). Take $\alpha$ the complement of the inclination of the radius vector to the tangent planethat is, $\alpha$ the inclination of the radius vector to the normal, or, what is the same thing, to the perpendicular from the origin on the tangent plane (whence, also, if $\mathrm{mp}$ be the length of this perpendicular, then $p=r \cos \alpha$ ). The shell-thickness in the direction of the radius vector is $=r d m$, or we have $d \nu=r d m \cos \alpha$; the element of potential is therefore $=\frac{d m}{m} \cos \alpha d \Sigma$. But $d \omega$ being the spherical aperture of the cone subtending the element $d \Sigma$, the perpendicular section at the distance $m r$ is $=m^{2} r^{2} d \omega$; we have therefore $d \Sigma=\frac{1}{\cos \alpha} m^{2} r^{2} d \omega$; and hence the element of potential is $=m d m \cdot r^{2} d \omega$, or the potential of the subtended portion of the shell is as before, $=m d m \int r^{2} d \omega$.

5. It may be added that, integrating between the values $m, n(m>n)$, we obtain $\frac{1}{2}\left(m^{2}-n^{2}\right) \int r^{2} d \omega$ for the potential of the shell-portion included between the surfaces $m r, n r$; and if $n=0$, then, as before, the potential of the cone is $=\frac{1}{2} m^{2} \int r^{2} d \omega$. 
Cone on a plane base, and plane figure.

6. Suppose that the surface $\Sigma$ is a plane; the surface $\Sigma^{\prime}$ is, of course, a parallel plane. Taking here $m p$ for the perpendicular distance of the plane $\Sigma$ from the origin, then, if $\delta$ be the infinitesimal distance of the two planes from each other, we have $\delta=p d m$, that is, $d m=\frac{\delta}{p}$; the potential of the cone is, as before, $=\frac{1}{2} m^{2} \int r^{2} d \omega$, and that of the plane figure, thickness $\delta$, is $=\frac{m \delta}{p} \int r^{2} d \omega$.

7. Taking, for greater convenience, $m=1$, we have

$$
\begin{aligned}
\text { Potential of cone } & =\frac{1}{2} \int r^{2} d \omega, \\
\text { Do. of plane figure } & =\frac{\delta}{p} \int r^{2} d \omega,
\end{aligned}
$$

where $p$ is now the perpendicular distance of the plane from the vertex; or if, as regards the plane figure, the infinitesimal thickness $\delta$ is taken as unity, then

$$
\text { Potential of plane figure }=\frac{1}{p} \int r^{2} d \omega .
$$

In each case $r$ is the value of the radius vector corresponding to a point of the plane figure which is the base of the cone, and the integration extends over the spherical aperture of the cone.

8. If the position of the radius vector is determined by the usual angular coordinates, $\theta$ its inclination to the axis of $z$, and $\phi$ its azimuth from the plane of $z x$-viz. if we have

$$
\begin{aligned}
& x=r \sin \theta \cos \phi \\
& y=r \sin \theta \sin \phi \\
& z=r \cos \theta
\end{aligned}
$$

then, as is well-known, $d \omega=\sin \theta d \theta d \phi$, and the integral $\int r^{2} d \omega$ is $=\int r^{2} \sin \theta d \theta d \phi$.

Taking the inclination of $p$ to the axes to be $\alpha, \beta, \gamma$ respectively, the equation of the plane which is the base of the cone is

$$
x \cos \alpha+y \cos \beta+z \cos \gamma=p
$$

viz. we have

$$
r[(\cos \alpha \cos \phi+\cos \beta \sin \phi) \sin \theta+\cos \gamma \cos \theta]=p
$$

that is,

$$
r=\frac{p}{(\cos \alpha \cos \phi+\cos \beta \sin \phi) \sin \theta+\cos \gamma \cos \theta}
$$


and the integral $\int r^{2} d \omega$ is therefore

$$
=p^{2} \int \frac{\sin \theta d \theta d \phi}{[(\cos \alpha \cos \phi+\cos \beta \sin \phi) \sin \theta+\cos \gamma \cos \theta]^{2}}
$$

and, in particular, if $p$ coincide with the axis of $z$, so that the equation of the plane is $z=p$, then the integral is

$$
=p^{2} \int \frac{\sin \theta d \theta d \phi}{\cos ^{2} \theta} .
$$

9. The integration in regard to $\theta$ can be at once performed; viz. in the latter case we have $\int \frac{\sin \theta d \theta}{\cos ^{2} \theta}=\sec \theta$; and in the former case, writing, as we may do,

then

$$
(\cos \alpha \cos \phi+\cos \beta \sin \phi) \sin \theta+\cos \gamma \cos \theta=M \cos (\theta-N),
$$

$$
\begin{gathered}
\int \frac{\sin \theta d \theta}{[(\cos \alpha \cos \phi+\cos \beta \sin \phi) \sin \theta+\cos \gamma \cos \theta]^{2}}=\frac{1}{M^{2}} \int \frac{\sin (\theta-N+N) d \theta}{\cos ^{2}(\theta-N)} \\
=\frac{1}{M^{2}}\left[\cos N \int \frac{\sin (\theta-N) d \theta}{\cos ^{2}(\theta-N)}+\sin N \int \frac{d \theta}{\cos (\theta-N)}\right] \\
=\frac{1}{M^{2}}\left[\cos N \sec (\theta-N)+\sin N \log \tan \left\{\frac{1}{4} \pi+\frac{1}{2}(\theta-N)\right\}\right] .
\end{gathered}
$$

\section{Case of a Polyhedron or a Polygon.}

10. Consider now the pyramid, vertex the origin $O$, standing on a polygonal base. Letting fall from the vertex a perpendicular $O M$ on the base of the pyramid, and drawing planes through $O M$ and the several vertices of the polygon, we thus divide the pyramid into triangular pyramids; viz. $A B$ being any side of the polygon, a component pyramid (or tetrahedron) will be $O M A B$, vertex $O$ and base $M A B$, where $M O$ is a perpendicular at $M$ to the triangular base $M A B$. And drawing through $M O$ a plane at right angles to $A B$, meeting it in $D$ (viz. $M D$ is the perpendicular from $M$ on the base $A B$ of the triangle), we divide the triangular pyramid into two pyramids $O M A D, O M B D$, each having for its base a right-angled triangle; viz. the vertex is $O$, the base is the triangle $A D M$ (or, as the case may be, $B D M$ ) right-angled at $D$, and $O M$ is a perpendicular at the vertex $M$ to the plane of the triangle. It is to be observed that, in speaking of the original pyramid as thus divided, we mean that the pyramid is the sum of the component pyramids taken each with the proper sign, + or - , as the case may be.

11. In the case of a polyhedron, this is in the like sense divisible into pyramids having for the common vertex the origin or point 0 , and standing on the several faces respectively; hence the polyhedron is ultimately divisible into triangular pyramids such as $O A D M$, where $A D M$ is a triangle right-angled at $D$, and where $O M$ is a perpendicular at $M$ to the plane of the triangle. Hence the potential of the polyhedron 
in regard to the point $O$ depends upon that of the pyramid $O A D M$; and (what is the same thing) the potential of any plane polygon in regard to the point $O$ depends upon that of the right-angled triangle $A D M$, situate as above in regard to the point $0 . I$

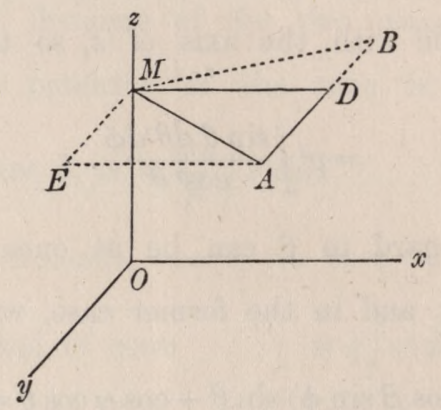

take $O M=h, M D=f, D A=g$; viz. supposing, as we may do, that the plane of the triangle is parallel to that of $x y$, the point $M$ on the axis of $z$, and the side $M D$ parallel to the axis of $x$, then $f, g, h$ will be the coordinates of the point $A$.

Formula for component triangular Pyramid, and Triangle.

12. Writing, as above, $x=r \sin \theta \cos \phi, y=r \sin \theta \sin \phi, z=r \cos \theta$, and observing that $h$ is the perpendicular distance originally called $p$, we have, for the potential of the pyramid,

$$
\begin{aligned}
V=\frac{1}{2} \int r^{2} d \omega & =\frac{1}{2} h^{2} \int \frac{\sin \theta d \theta d \phi}{\cos ^{2} \theta} \\
& =\frac{1}{2} h^{2} \int d \phi(\sec \theta)
\end{aligned}
$$

where, $\phi$ being regarded as a given angle, the invegral expression $\sec \theta$ must be taken from $\theta=0$ to the value of $\theta$ corresponding to a point in the side $A D$. For any such point we have $f=r \sin \theta \cos \phi, h=r \cos \theta$, that is, $\frac{f}{h}=\tan \theta \cos \phi$, or the required value of $\theta$ is $=\tan ^{-1} \frac{f}{h \cos \phi}$, and consequently that of $\sec \theta$ is

$$
\sqrt{1+\frac{f^{2}}{h^{2} \cos ^{2} \phi}},=\frac{1}{h \cos \phi} \sqrt{f^{2}+h^{2} \cos ^{2} \phi}
$$

or, as this may also be written,

hence

$$
=\frac{1}{h} \sqrt{f^{2}+h^{2}+f^{2} \tan ^{2} \phi}
$$

$$
V=\frac{1}{2} h \int\left(\sqrt{f^{2}+h^{2}+f^{2} \tan ^{2} \phi}-h\right) d \phi
$$


13. The first term of the integral, writing therein for a moment $\tan \phi=x$, is

$$
\begin{gathered}
\int \frac{\left(f^{2}+h^{2}+f^{2} x^{2}\right) d x}{\left(1+x^{2}\right) \sqrt{f^{2}+h^{2}+f^{2} x^{2}}} \\
=f^{2} \int \frac{d x}{\sqrt{f^{2}+h^{2}+f^{2} x^{2}}}+h^{2} \int \frac{d x}{\left(1+x^{2}\right) \sqrt{f^{2}+h^{2}+f^{2} x^{2}}} \\
=f \log \left(f x+\sqrt{f^{2}+h^{2}+f^{2} x^{2}}\right)+h \tan ^{-1} \frac{h x}{\sqrt{f^{2}+h^{2}+f^{2} x^{2}}} .
\end{gathered}
$$

Hence, replacing $x$ by its value, we have

$$
V=\frac{1}{2} h\left\{h \tan ^{-1} \frac{h \tan \phi}{\sqrt{f^{2}+h^{2}+f^{2} \tan ^{2} \phi}}+f \log \left(f \tan \phi+\sqrt{f^{2}+h^{2}+f^{2} \tan ^{2} \phi}\right)-h \phi\right\},
$$

to be taken from $\phi=0$ to the value of $\phi$ corresponding to the point $A$; viz. we have here $f=r \sin \theta \cos \phi, g=r \sin \theta \sin \phi, h=r \cos \theta$, and thence $\tan \phi=\frac{g}{f}$ or $f \tan \phi=g$; whence, writing for shortness, $s=\sqrt{f^{2}+g^{2}+h^{2}}$ (viz. $s$ denotes the distance $O A$ ), we have

or, observing that

$$
V=\frac{1}{2} h\left\{h \tan ^{-1} \frac{g h}{f s}+f \log \frac{s+g}{\sqrt{f^{2}+h^{2}}}-h \tan ^{-1} \frac{g}{f}\right\}
$$

this is

$$
\frac{s+g}{s-g}=\frac{(s+g)^{2}}{\sqrt{f^{2}+h^{2}}}
$$

$$
V=\frac{1}{2} h\left\{h \tan ^{-1} \frac{g h}{f s}+\frac{1}{2} f \log \frac{s+g}{s-g}-h \tan ^{-1} \frac{g}{f}\right\},
$$

for the potential of the pyramid $O M D A$ in regard to the point $O$; by omitting the factor $\frac{1}{2} h$, we have

$$
V=h \tan ^{-1} \frac{g h}{f s}+\frac{1}{2} f \log \frac{s+g}{s-g}-h \tan ^{-1} \frac{g}{f}
$$

for the potential of the triangle $M D A$. The expression $\tan ^{-1}$ denotes, here and elsewhere, an arc included between the limits $-\frac{\pi}{2},+\frac{\pi}{2}$ : it is therefore + or - according as the tangent is + or - .

Formulce for rectangular Pyramid, and Rectangle.

14. Completing the rectangle $M D A E$, the potential of the triangle $A M E$ is obtained by interchanging the letters $g$ and $f$; viz. we have

$$
V=h \tan ^{-1} \frac{f h}{g s}+\frac{1}{2} g \log \frac{s+f}{s-f}-h \tan ^{-1} \frac{f}{g}
$$

for the potential of the triangle $M E A$. 
The sum of the two gives the potential of the rectangle $M D A E$; viz. for this rectangle, we have

But we have

$$
V=h\left(\tan ^{-1} \frac{g h}{f s}+\tan ^{-1} \frac{f h}{g s}-\frac{\pi}{2}\right)+\frac{1}{2} f \log \frac{s+g}{s-g}+\frac{1}{2} g \log \frac{s+f}{s-f} .
$$

$$
\tan ^{-1} \frac{g h}{f s}+\tan ^{-1} \frac{h f}{g s}+\tan ^{-1} \frac{f g}{h s}=\frac{\pi}{2}
$$

for the function on the left hand is

$$
=\tan ^{-1} \frac{\frac{g h}{f s}+\frac{h f}{g s}+\frac{f g}{h s}-\frac{f g h}{s^{3}}}{1-\frac{f^{2}}{s^{2}}-\frac{g^{2}}{s^{2}}-\frac{h^{2}}{s^{2}}}
$$

viz. the denominator being $1-\frac{f^{2}+g^{2}+h^{2}}{s^{2}},=0$, the tangent of the arc is $\infty$, and the component arcs being each positive and less than $\frac{\pi}{2}$, the arc in question can only be $=\frac{\pi}{2}$. We have consequently

$$
V=-h \tan ^{-1} \frac{f g}{h s}+\frac{1}{2} f \log \frac{s+g}{s-g}+\frac{1}{2} g \log \frac{s+f}{s-f}
$$

for the potential of the rectangle $M D A E$. And, multiplying this by $\frac{1}{2} h$, we have

$$
V=-\frac{1}{2} h^{2} \tan ^{-1} \frac{f g}{h s}+\frac{1}{4} h f \log \frac{s+g}{s-g}+\frac{1}{4} g h \log \frac{s+f}{s-f}
$$

for the potential of the rectangular pyramid, vertex $O$ and base $M D A E$.

\section{Formula for the Cuboid.}

15. Completing the rectangular parallelopiped, or, say for shortness, the "cuboid," the sides whereof are $(f, g, h)$; this breaks up into three pyramids, standing on the rectangles $f g, g h$, and $h f$ respectively; and the potentials for the last two pyramids are at once obtained from the last-mentioned expression of $V$ by mere cyclical interchanges of the letters. Adding the three expressions, we obtain

$$
V=\frac{1}{2} g h \log \frac{s+f}{s-f}+\frac{1}{2} h f \log \frac{s+g}{s-g}+\frac{1}{2} f g \log \frac{s+h}{s-h}-\frac{1}{2} f^{2} \tan ^{-1} \frac{g h}{f s}-\frac{1}{2} g^{2} \tan ^{-1} \frac{h f}{g s}-\frac{1}{2} h^{2} \tan ^{-1} \frac{f g}{h s}
$$

for the potential of the cuboid.

Group of Results, for Point, Line, Rectangle, and Cuboid.

16. It is convenient to prefix two results, that for the potential of the point $A$ (mass taken to be unity), and that for the potential of the line $A E$ (density taken to 
be unity, or mass of an element of length $d x$, taken to be $=d x$ ). We have, the attracted point being always at $O$,

$$
\begin{aligned}
& \text { Potential of point } A \quad=\frac{1}{s}, \quad\left(s=\sqrt{f^{2}+g^{2}+h^{2}} \text {, as before }\right) \text {, } \\
& \text { Potential of line } A E \quad=\frac{1}{2} \log \frac{s+f}{s-f} \text {, } \\
& \text { Potential of rectangle } M D A E=\frac{1}{2} g \log \frac{s+f}{s-f}+\frac{1}{2} f \log \frac{s+g}{s-g}-h \tan ^{-1} \frac{f g}{h s} \text {, } \\
& \text { Potential of cuboid } \quad=\frac{1}{2} g h \log \frac{s+f}{s-f}+\frac{1}{2} h f \log \frac{s+g}{s-g}+\frac{1}{2} f g \log \frac{s+h}{s-h} \\
& -\frac{1}{2} f^{2} \tan ^{-1} \frac{g h}{f s}-\frac{1}{2} g^{2} \tan ^{-1} \frac{h f}{g s}-\frac{1}{2} h^{2} \tan ^{-1} \frac{f g}{h s},
\end{aligned}
$$

which functions may be called $A(f, g, h), B(f, g, h), C(f, g, h)$, and $D(f, g, h)$ respectively. It is to be observed that $f, g, h$ are taken to be each of them positive, and that $s$ denotes in every case the positive value of $\sqrt{f^{2}+g^{2}+h^{2}}$; for a symmetrically situated body, corresponding to negative values of each or any of these quantities, the potential has in each case its original value, without change of sign. But $B$ is an odd function as regards $f, C$ an odd function as regards $f$ or $g, D$ an odd function as regards $f, g$, or $h$; for example, $C(-f, g, \pm h)$ and $C(f,-g, \pm h)$ are each $=-C(f, g, h)$, and therefore of course $C(-f,-g, \pm h)=C(f, g, h)$.

\section{Extension to case where the attracted point has an arbitrary position.}

17. The attracted point has thus far been considered as in a definite position in regard to the attracting mass; but it is easy to pass to the general case of any relative position whatever. Thus, for a line $A B$, if $M$ be the foot of the perpendicular let fall from the point $O$, and if, to fix the ideas, the order of succession of the three points is $A, B, M$, then, with respect to the point $O$,

$$
\text { line } A B=\text { line } A M-\text { line } B M \text {. }
$$

$$
\text { A } \quad B \quad M
$$

Taking the $y$ - and $z$-coordinates to be $b, c$, the $x$-coordinates for the points $A, B, M$ to be $x_{0}, x_{1}, a$ respectively, and in the figure $a>x_{1}, x_{1}>x_{0}$, then $a-x_{0}, a-x_{1}$ are each of them positive, $a-x_{0}$ being the greater, the potential of the line $A M$ is $=B\left(a-x_{0}, b, c\right)$, that of $B M$ is $=B\left(a-x_{1}, b, c\right)$, and the potential of the whole line is

$$
=B\left(a-x_{0}, b, c\right)-B\left(a-x_{1}, b, c\right) ;
$$

viz. this formula is proved for the case where $M$ is situate as in the figure. But supposing that $A$ and $B$ retain their relative position (viz. $x_{1}>x_{0}$ ), then the formula holds good for any other position of $M$; thus, if $M$ be between the points $A, B$ viz. if the order is $A, M, B$-then

$$
\text { line } A B=\text { line } A M+\text { line } B M \text {, }
$$

C. IX. 
and potential is

$$
=B\left(a-x_{0}, b, c\right)+B\left(x_{1}-a, b, c\right),
$$

where the second term is $=-B\left(a-x_{1}, b, c\right)$; and so, if the order is $M, A, B$, then

$$
\text { line } A B=\text { line } B M-\text { line } A M \text {, }
$$

and the potential is $B\left(x_{1}-a, b, c\right)-B\left(x_{0}-a, b, c\right)$, which is

$$
=-B\left(a-x_{1}, b, c\right)+B\left(a-x_{0}, b, c\right) \text {. }
$$

18. Similarly for a rectangle $A B C D$, if $M$, the foot of the perpendicular from the point $O$, has the position shown in the figure, then

$$
\text { rectangle } \begin{aligned}
A D= & \text { rectangle } M C \\
& - \text { rectangle } M A \\
& - \text { rectangle } M D \\
& + \text { rectangle } M B
\end{aligned}
$$

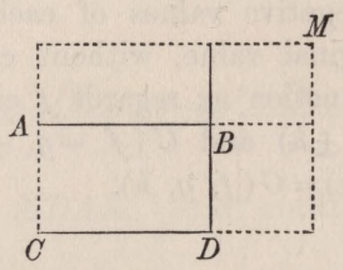

where $O$ is a point on the perpendicular at the common vertex $M$ of the four rectangles; and the resulting expression for the rectangle $A D$ will apply to any position of the point $M$.

19. And in like manner for a cuboid; taking the point $O$ in any determinate position, the cuboid may be decomposed into eight cuboids (each with the sign + or as the case may be) having the point $O$ for a common vertex; and the resulting expression for the potential will apply to any position whatever of the point $M$.

20. The results may be collected and exhibited as follows:-the coordinates of the attracted point are $a, b, c$; and it is assumed that $x_{1}>x_{0}, y_{1}>y_{0}, z_{1}>z_{0}$, (viz. for $x$ the order is $+\infty, x_{1}, x_{0},-\infty$, and so for $y$ and $z$ respectively).

Potential of point $(x, y, z)$

$$
\begin{aligned}
\text { is }= & A(a-x, b-y, c-z) ; \\
\text { is }= & B\left(a-x_{0}, b-y, c-z\right) \\
& -B\left(a-x_{1}, b-y, c-z\right) ;
\end{aligned}
$$

Potential of line $\left(x_{1}, y, z\right),\left(x_{0}, y, z\right)$

Potential of rectangle $\left(x_{1}, y_{1}, z\right),\left(x_{0}, y_{1}, z\right)$ is $=C\left(a-x_{0}, b-y_{0}, c-z\right)$

$$
\begin{aligned}
\left(x_{1}, y_{0}, z\right),\left(x_{0}, y_{0}, z\right) & -C\left(a-x_{0}, b-y_{1}, c-z\right) \\
& -C\left(a-x_{1}, b-y_{0}, c-z\right) \\
& +C\left(a-x_{1}, b-y_{1}, c-z\right)
\end{aligned}
$$


Potential of cuboid

$$
\begin{aligned}
\left(x_{1}, y_{1}, z_{1}\right),\left(x_{0}, y_{1}, z_{1}\right) \text { is }= & D\left(a-x_{0}, b-y_{0}, c-z_{0}\right) \\
\left(x_{1}, y_{0}, z_{1}\right),\left(x_{0}, y_{0}, z_{1}\right) & -D\left(a-x_{1}, b-y_{0}, c-z_{0}\right) \\
\left(x_{1}, y_{1}, z_{0}\right),\left(x_{0}, y_{1}, z_{0}\right) & -D\left(a-x_{0}, b-y_{1}, c-z_{0}\right) \\
\left(x_{1}, y_{0}, z_{0}\right),\left(x_{0}, y_{0}, z_{0}\right) & +D\left(a-x_{1}, b-y_{1}, c-z_{0}\right) \\
& -D\left(a-x_{0}, b-y_{0}, c-z_{1}\right) \\
& +D\left(a-x_{1}, b-y_{0}, c-z_{1}\right) \\
& +D\left(a-x_{0}, b-y_{1}, c-z_{1}\right) \\
& -D\left(a-x_{1}, b-y_{1}, c-z_{1}\right) .
\end{aligned}
$$

21. These are connected together as follows, viz.:-

$$
\begin{aligned}
& \text { Potential of line } \quad=\int_{x_{0}}^{x_{1}} d x \text { Potential of point, } \\
& \text { Potential of rectangle }=\int_{y_{0}}^{y_{1}} d y \text { Potential of line, } \\
& \text { Potential of cuboid }=\int_{z_{0}}^{z_{1}} d z \text { Potential of rectangle, }
\end{aligned}
$$

equations which are in fact of the form

$$
\begin{aligned}
& B(x, y, z)=\int d x A(x, y, z), \\
& C(x, y, z)=\int d y B(x, y, z), \\
& D(x, y, z)=\int d z C(x, y, z) .
\end{aligned}
$$

Differential properties of the functions $A, B, C, D$.

22. These relations, with other allied ones, may be verified as follows. Writing $r=\sqrt{x^{2}+y^{2}+z^{2}}$, the fundamental forms are

$$
\log \frac{r+x}{r-x}, \text { and } \tan ^{-1} \frac{y z}{r x} .
$$

We have $d_{x} r=\frac{x}{r}$, \&c., and thence

$$
\begin{aligned}
& d_{x} \log \frac{r+x}{r-x}=\frac{1+\frac{x}{r}}{r+x}-\frac{-1+\frac{x}{r}}{r-x},=\frac{1}{r}+\frac{1}{r},=\frac{2}{r} \\
& d_{y} \log \frac{r+x}{r-x}=\frac{\frac{y}{r}}{r+x}-\frac{\frac{y}{r}}{r-x},=\frac{y}{r}\left(\frac{1}{r+x}-\frac{1}{r-x}\right),=\frac{-2 x y}{r\left(r^{2}-x^{2}\right)}
\end{aligned}
$$


276

ON THE POTENTIALS OF POLYGONS AND POLYHEDRA.

$[602$

or, since

this is

$$
r^{2}+x^{2}=\left(r^{2}-y^{2}\right)+\left(r^{2}-z^{2}\right) \text { and } r^{2} x^{2}+y^{2} z^{2}=\left(r^{2}-y^{2}\right)\left(r^{2}-z^{2}\right),
$$

$$
=-\frac{y z}{r}\left(\frac{1}{r^{2}-y^{2}}+\frac{1}{r^{2}-z^{2}}\right)
$$

$$
d_{y} \tan ^{-1} \frac{y z}{r x}=\frac{r x \cdot z-y z \cdot \frac{x y}{r}}{r^{2} x^{2}+y^{2} z^{2}}=\frac{x z}{r} \frac{r^{2}-y^{2}}{r^{2} x^{2}+y^{2} z^{2}},
$$

which, the denominator being, as before, $\left(r^{2}-y^{2}\right)\left(r^{2}-z^{2}\right)$, is

$$
=\frac{x z}{r} \frac{1}{r^{2}-z^{2}} \text {. }
$$

It is now easy to form the following results:-

23. First,

$$
\begin{aligned}
u & =A(x, y, z)=\frac{1}{r} \text { (symmetrical), } \\
d_{x} u & =-\frac{x}{r^{3}}, \& c ., \\
d_{x}{ }^{2} u & =\frac{3 x^{2}}{r^{5}}-\frac{1}{r^{3}}, \& c ., d_{x} d_{y} u=\frac{3 x y}{r^{5}}, \& c .,
\end{aligned}
$$

and thence

$$
\left(d_{x}^{2}+d_{y}^{2}+d_{z}^{2}\right) u=0
$$

24. Secondly,

$$
u=B(x, y, z)=\frac{1}{2} \log \frac{r+x}{r-x}(\text { symmetrical as to } y, z) ;
$$

then

$$
\begin{aligned}
& d_{x} u=\frac{1}{r}(=A(x, y, z)), \quad d_{y} u=\frac{-x y}{r\left(r^{2}-x^{2}\right)}, \quad \& c . \\
& d_{x}^{2} u=-\frac{x}{r^{3}}, \quad d_{x} d_{y} u=-\frac{y}{r^{3}}, \quad d_{y} d_{z} u=\frac{x y z}{r^{3}\left(r^{2}-x^{2}\right)}+\frac{2 x y z}{r\left(r^{2}-x^{2}\right)^{2}}, \& c . \\
& d_{y}^{2} u=\frac{-x}{r\left(r^{2}-x^{2}\right)}+\frac{2 x y^{2}}{r\left(r^{2}-x^{2}\right)^{2}}+\frac{x y^{2}}{r^{3}\left(r^{2}-x^{2}\right)}, \& c .
\end{aligned}
$$

and thence

$$
\left(d_{x}^{2}+d_{y}^{2}+d_{z}^{2}\right) u=-\frac{x}{r^{3}}-\frac{2 x}{r\left(r^{2}-x^{2}\right)}+\frac{2 x}{r\left(r^{2}-x^{2}\right)}+\frac{x}{r^{3}},=0 .
$$

25. Thirdly,

$$
\begin{aligned}
u & \left.=C(x, y, z)=\frac{1}{2} y \log \frac{r+x}{r-x}+\frac{1}{2} x \log \frac{r+y}{r-y}-z \tan ^{-1} \frac{x y}{z r} \text { (symmetrical as to } x, y\right) \\
d_{y} u & =\frac{1}{2} \log \frac{r+x}{r-x}(=B(x, y, z)):
\end{aligned}
$$

www.rcin.org.pl 
in verification whereof, observe that the remaining terms are

which is $=0$;

$$
\begin{aligned}
& =-\frac{x y^{2}}{r \cdot r^{2}-x^{2}}+\frac{x}{r}-\frac{x z^{2}}{r} \frac{r^{2}-y^{2}}{\left(r^{2}-x^{2}\right)\left(r^{2}-y^{2}\right)}, \\
& =\frac{x}{r}\left(-\frac{y^{2}}{r^{2}-x^{2}}+1-\frac{z^{2}}{r^{2}-x^{2}}\right), \\
& =\frac{x}{r\left(r^{2}-x^{2}\right)}\left(-y^{2}+r^{2}-x^{2}-z^{2}\right),
\end{aligned}
$$

$$
\begin{aligned}
d_{z} u & =-\frac{x y z}{r\left(r^{2}-x^{2}\right)}-\frac{x y z}{r\left(r^{2}-y^{2}\right)}+\frac{x y z}{r} \frac{r^{2}-x^{2}+r^{2}-y^{2}}{\left(r^{2}-x^{2}\right)\left(r^{2}-y^{2}\right)}-\tan ^{-1} \frac{x y}{z r} \\
& =-\tan ^{-1} \frac{x y}{z r} \\
d_{x}^{2} u & =-\frac{x y}{r\left(r^{2}-y^{2}\right)}, \\
d_{z}^{2} u & =\frac{x y}{r} \frac{r^{2}-x^{2}+r^{2}-y^{2}}{\left(r^{2}-x^{2}\right)\left(r^{2}-y^{2}\right)}, \quad=\frac{x y}{r}\left(\frac{1}{r^{2}-x^{2}}+\frac{1}{r^{2}-y^{2}}\right)
\end{aligned}
$$

and thence

$$
\left(d_{x}^{2}+d_{y}^{2}+d_{z}^{2}\right) u=0
$$

26. Fourthly,

$$
\begin{aligned}
& u=D(x, y, z)=\frac{1}{2} y z \log \frac{r+x}{r-x}+\frac{1}{2} z x \log \frac{r+y}{r-y}+\frac{1}{2} x y \log \frac{r+z}{r-z}-\frac{1}{2} x^{2} \tan ^{-1} \frac{y z}{x r}-\frac{1}{2} y^{2} \tan ^{-1} \frac{z x}{y r}-\frac{1}{2} z^{2} \tan ^{-1} \frac{x y}{z r} \text { (symmetrical), } \\
& d_{z} u=\frac{1}{2} y \log \frac{r+x}{r-x}+\frac{1}{2} x \log \frac{r+y}{r-y}-z \tan ^{-1} \frac{x y}{z r}=C(x, y, z) \\
& d_{z}^{2} u=-\tan ^{-1} \frac{x y}{z r}
\end{aligned}
$$

and thence

$$
\begin{aligned}
\left(d_{x}^{2}+d_{y}{ }^{2}+d_{z}^{2}\right) u & =-\tan ^{-1} \frac{y z}{x r}-\tan ^{-1} \frac{z x}{y r}-\tan ^{-1} \frac{x y}{z r} \\
& =-\tan ^{-1} \frac{\frac{y z}{x r}+\frac{z x}{y r}+\frac{x y}{z r}-\frac{x y z}{r^{3}}}{1-\frac{x^{2}}{r^{2}}-\frac{y^{2}}{r^{2}}-\frac{z^{2}}{r^{2}}}
\end{aligned}
$$

viz. the denominator being $=0$, the arc is $\pm \frac{\pi}{2}$, or we have

$$
\left(d_{x}^{2}+d_{y}^{2}+d_{z}^{2}\right) u=\mp \frac{\pi}{2}
$$

the value being $-\frac{\pi}{2}$ if $x, y, z$ are all three of them, or only one, positive; but $+\frac{\pi}{2}$ if they are all three of them, or only one, negative. 


\section{Application to the Potentials of the Point, the Line, the Rectangle, and the Cuboid.}

27. Take now $V$ to denote in succession the foregoing expressions of the potential of a point, a line, a rectangle, or a cuboid, at the point $(a, b, c)$. In the first three cases respectively, each of the component terms is reduced to zero by the operator $d_{a}^{2}+d_{b}{ }^{2}+d_{c}{ }^{2} ;$ and we have, therefore,

$$
\left(d_{a}^{2}+d_{b}^{2}+d_{c}^{2}\right) V=0,
$$

which is as it should be. But in the case of the cuboid, each of the eight component terms is by the operator reduced to $\mp \frac{\pi}{2}$, and we have therefore

$$
\left(d_{a}^{2}+d_{b}^{2}+d_{c}^{2}\right) V=\Sigma\left\{ \pm\left(\mp \frac{\pi}{2}\right)\right\}
$$

$\Sigma$ denoting the sum of eight terms, the \pm denoting + or - , according to the sign of the term in the formula (viz. in four cases this is + , and in four cases it is -), and the $\mp \frac{\pi}{2}$ denoting the value $\frac{\pi}{2}$ with its proper sign depending on the signs of the quantities $\left(a-x_{0}, b-y_{0}, c-z_{0}\right)$, \&c., as explained in the preceding Number.

Suppose for a moment $a>x_{1}, b>y_{1}, c>z_{1}$, or the attracted point in one of the regions exterior to the cuboid; then $\mp \frac{\pi}{2}$ will in each case be $=-\frac{\pi}{2}$, and the sign $\mp$, being + for four of the terms and - for the four remaining terms, the sum is $=0$. And similarly, in all cases where the attracted point is exterior to the cuboid, the sum of the eight terms is $=0$. But when the attracted point is interior, that is, when $a>x_{0}<x_{1}, b>y_{0}<y_{1}, c>z_{0}<z_{1}$, then it is found that, for the four terms which have the sign + , the value of $\mp \frac{\pi}{2}$ is $=-\frac{\pi}{2}$; and for the four terms which have the sign - , its value is $=+\frac{\pi}{2}$; whence, in the sum, vach term is $=-\frac{\pi}{2}$, or the value is $=-4 \pi$. Hence, in the case of the cuboid, we have

$$
\left(d_{a}^{2}+d_{b}^{2}+d_{c}^{2}\right) V=0 \text { or }-4 \pi,
$$

according as the attracted point is external or internal.

\section{Verification in regard to the Rectangle.}

28. I start from the formula

$$
\begin{aligned}
V= & C\left(a-x_{0}, b-y_{0}, c\right) \\
& -C\left(a-x_{1}, b-y_{0}, c\right) \\
& -C\left(a-x_{0}, b-y_{1}, c\right) \\
& +C\left(a-x_{1}, b-y_{1}, c\right)
\end{aligned}
$$


where, as before, $x_{1}>x_{0}, y_{1}>y_{0} . \quad V$ is here a function of $(a, b, c)$, satisfying the partial differential equation

$$
\left(d_{a}^{2}+d_{b}^{2}+d_{c}^{2}\right) V=0
$$

and (as is easily verified) vanishing when any one of the variables $a, b, c$ becomes infinite; it does not become infinite for any finite values of $a$ or $b$, or any positive value of $c$. Hence, by a theorem of Green's*, there exists on the plane $z=0$ a distribution of matter giving rise to the potential $V$; and not only so, but the density at any point $(x, y)$ of the plane is given by the formula

$$
\rho=-\frac{1}{2 \pi}\left(\frac{d W}{d c}\right)_{c=0}
$$

where $W$ is what $V$ becomes on writing therein $x, y$ in place of $a, b$, and $c=0$ is regarded as an indefinitely small positive quantity.

We have

$$
d_{c} C(x, y, c)=-\tan ^{-1} \frac{x y}{c r} \text {, where } r=\sqrt{x^{2}+y^{2}+c^{2}}
$$

And hence

$$
\begin{aligned}
d_{c} W= & -\tan ^{-1} \frac{\left(x-x_{0}\right)\left(y-y_{0}\right)}{c \sqrt{\left(x-x_{0}\right)^{2}+\left(y-y_{0}\right)^{2}+c^{2}}} \\
& +\tan ^{-1} \frac{\left(x-x_{1}\right)\left(y-y_{0}\right)}{c \sqrt{\left(x-x_{1}\right)^{2}+\left(y-y_{0}\right)^{2}+c^{2}}} \\
& +\tan ^{-1} \frac{\left(x-x_{0}\right)\left(y-y_{1}\right)}{c \sqrt{\left(x-x_{0}\right)^{2}+\left(y-y_{1}\right)^{2}+c^{2}}} \\
& -\tan ^{-1} \frac{\left(x-x_{1}\right)\left(y-y_{1}\right)}{c \sqrt{\left(x-x_{1}\right)^{2}+\left(y-y_{1}\right)^{2}+c^{2}}}
\end{aligned}
$$

Putting $c=0$, as above, each arc is $=\frac{\pi}{2}$ or $-\frac{\pi}{2}$, according as the fraction under the $\tan ^{-1}$ is positive or negative-that is, according as the numerator is positive or negative. Suppose for a moment $x>x_{1}, y>y_{1}$, viz. the point $(x, y)$ is here in a region exterior to the rectangle $\left(x_{1}, y_{1}\right),\left(x_{1}, y_{0}\right),\left(x_{0}, y_{1}\right),\left(x_{0}, y_{0}\right)$ : the value of $d_{c} W$ is $=-\frac{\pi}{2}+\frac{\pi}{2}+\frac{\pi}{2}-\frac{\pi}{2},=0$; and similarly, for every other position of the point $(x, y)$

* The theorem in question is a particular case of Green's, $4 \pi \rho=-\frac{d V}{d w}-\frac{d V^{\prime}}{d w}$ ("Essay on the Application of Mathematical Analysis to the Theories of Electricity and Magnetism" (1828), see p. 31 of the Collected Works); viz. the surface is here a plane, and $\boldsymbol{V}=V^{\prime}$. And it is also a particular case of the formula $\rho^{\prime}=\frac{-\Gamma_{2}^{\frac{1}{2}}(n-1)}{2 \pi^{\frac{1}{2} s} \Gamma_{\frac{1}{2}}(n-s+1)} P^{\prime}$ (" Memoir on the Determination of the Exterior and Interior Attraction of Ellipsoids of Variable Densities" (1835), see p. 199 of the Collected Works); viz. $s$ is taken =2; and Green's extraspatial coordinate $u$ then becomes the coordinate $z$ of ordinary tri-dimensional space. 
exterior to the rectangle, the value is $=0$. But for a point interior to the rectangle, we have $x<x_{1}>x_{0}, y<y_{1}>y_{0}$, and in this case the value is

$$
-\frac{\pi}{2}+\left(-\frac{\pi}{2}\right)+\left(-\frac{\pi}{2}\right)-\frac{\pi}{2},=-2 \pi
$$

Hence

$$
\rho,=-\frac{1}{2 \pi}\left(d_{c} W\right)_{c=0}, \text { is }=0 \text { or } 1,
$$

according as the point is exterior or interior to the rectangle, viz. the distribution producing the potential in question is a uniform distribution (density unity) over the rectangle, which is as it should be.

\section{Potential of a Cuboidal Surface.}

29. The preceding formulæ lead to the expression of the potential of a cuboidal surface (viz. the surface composed of the six faces of a cuboid, each of them being considered as a plate of the same uniform density) upon a point $a, b, c$. Writing, for convenience,

$$
\begin{aligned}
E(f, g, h)=\frac{1}{2}(g+h) \log \frac{s+f}{s-f}+\frac{1}{2}(h+f) \log & \frac{s+g}{s-g}+\frac{1}{2}(f+g) \log \left(\frac{s+h}{s-h}\right) \\
& -f \tan ^{-1} \frac{g h}{f s}-g \tan ^{-1} \frac{h f}{g s}-h \tan ^{-1} \frac{f g}{h s},
\end{aligned}
$$

where each term is supposed to have (compounded with its expressed sign) a sign \pm , as follows: viz. in any $f g \operatorname{term}\left(\frac{1}{2} f \log \frac{s+g}{s-g}, \frac{1}{2} g \log \frac{s+f}{s-f}\right.$, or $\left.h \tan ^{-1} \frac{f g}{h s}\right)$, this sign \pm is + if $f$ and $g$ are both positive or both negative, but is - if $f$ and $g$ are the one of them positive and the other negative; and the like as to the gh terms and the $h f$ terms respectively. And this being so, the expression for the potential (applying as well to an interior as to an exterior point) is

$$
\begin{aligned}
V= & E\left(a-x_{0}, b-y_{0}, c-z_{0}\right) \\
& +E\left(a-x_{1}, b-y_{0}, c-z_{0}\right) \\
& +E\left(a-x_{0}, b-y_{1}, c-z_{0}\right) \\
& +E\left(a-x_{1}, b-y_{1}, c-z_{0}\right) \\
& +E\left(a-x_{0}, b-y_{0}, c-z_{1}\right) \\
& +E\left(a-x_{1}, b-y_{0}, c-z_{1}\right) \\
& +E\left(a-x_{0}, b-y_{1}, c-z_{1}\right) \\
& +E\left(a-x_{1}, b-y_{1}, c-z_{1}\right)
\end{aligned}
$$

It is, in fact, easy to verify that the final result, interpreted as above, represents the sum of the six positive values, which are the values of the potential for the six faces of the cuboid respectively. 